\title{
A Tracking Differentiator Based Backstepping Control of Missile System
}

\section{Wenguang Zhang ${ }^{\text {a }}$, Lili Yang, Junwei Lei}

Department of Control, Naval aeronautical and astronautical university,Yantai, 264001,China

aemail: zhangwenguang1024@126.com

Keywords: Tracking differentiator; stability; Backstepping; Missile; Control system

\begin{abstract}
To avoid the complex calculation of derivative in the backstepping design procedure, a novel kind of tracking differentiator was adopt to solve the derivative of ideal angle speed of the pitch channel missile system. Also, the ideal angle speed should be bounded or the stability of the system can not be guaranteed. And a Lyapunov function was chosen to prove the stability of the whole system. At last, the detailed numerical simulation was done to show the rightness and effectiveness of the proposed method.
\end{abstract}

\section{Introduction}

Pitch channel of missile system can be reduced and simplified to be a second order system[1-4], which is a classical method to design and analysis of the stability of missile system. And backstepping method is a widely used method because the stability of the whole system can be guaranteed by choosing a Lyapunov energy function. But the only disadvantage is that the derivative of desired value is very complex[5-8]. So a kind of tracking differentiator method was widely studied to solve derivative of some signals. But there is no difference between tracking differentiator method and traditional filter method that both need the input signal is bound or its derivative is bound. So we design a kind of tracking differentiator based backstepping controller for missile pitch channel system, and simulation shows that strategy is very effective.

\section{Problem Description}

The simplified linear model of supersonic missile pitch channel can be written as following second order system:

$$
\begin{aligned}
& \dot{\alpha}=\omega_{z}-a_{34} \alpha-a_{35} \delta_{z}+f_{1} \\
& \dot{\omega}_{z}=a_{24} \alpha+a_{22} \omega_{z}+a_{25} \delta_{z}+f_{2}
\end{aligned}
$$

Where $a_{i j}$ is air dynamic coefficient of missile, $\alpha$ is attack angle of missile, $\omega_{z}$ is the rotate speed of pitch angle , $f_{1}$ and $f_{2}$ are outer disturbance.

The control objective is to design a backstepping control law $\delta_{z}$ based on tracking differentiator such that the attack angle $\alpha$ can track the desired angle $\alpha^{d}$. Without loss of generality, assume $\alpha^{d}=1$, and the disturbance $f_{1}$ and $f_{2}$ can be observed and compensated by the designed control law $\delta_{z}$.

\section{Backstepping Controller Design}

Define a new error variable as $e_{\alpha}=\alpha-\alpha^{d}$, then the first subsystem can be described as

$$
\dot{e}_{\alpha}=\omega_{z}-a_{34} \alpha-a_{35} \delta_{z}+f_{1}-\dot{\alpha}_{d}
$$

Then the idea value of $\omega_{z}$ can be designed as $\omega_{z}^{d}$ as follows:

$$
\omega_{z}^{d}=a_{34} \alpha+a_{35} \delta_{z}-f_{1}+\dot{\alpha}_{d}-k_{\alpha_{1}} e_{\alpha}-k_{\alpha 2} \frac{e_{\alpha}}{\left|e_{\alpha}\right|+\varepsilon_{b}}
$$

Since $f_{1}$ is unknown, we define a new variable as 


$$
\omega_{z a}^{d}=a_{34} \alpha+a_{35} \delta_{z}+\dot{\alpha}_{d}-k_{\alpha_{1}} e_{\alpha}-k_{\alpha 2} \frac{e_{\alpha}}{\left|e_{\alpha}\right|+\varepsilon_{b}}
$$

Then

$$
\omega_{z}^{d}=\omega_{z a}^{d}-f_{1}, \dot{\omega}_{z}^{d}=\dot{\omega}_{z a}^{d}-\dot{f}_{1}
$$

And Define a new variable as $e_{\omega}=\omega-\omega_{z}^{d}$, then the second subsystem can be rewritten as

$$
\dot{e}_{\omega}=a_{24} \alpha+a_{22} \omega_{z}+a_{25} \delta_{z}+f_{2}-\dot{\omega}_{z}^{d}
$$

It can also be rewritten as

$$
\dot{e}_{\omega}=a_{24} \alpha+a_{22} \omega_{z}+a_{25} \delta_{z}+f_{2}+\dot{f}_{1}-\dot{\omega}_{z a}^{d}
$$

And we design a tracking differentiator to solve $\dot{\omega}_{z a}^{d}$ as

$$
\begin{aligned}
& z_{1}=x_{1}-\omega_{z a}^{d} \\
& \dot{x}_{1}=x_{2} \\
& \dot{x}_{2}=R^{2}\left(-z_{1}-x_{2} / R\right)
\end{aligned}
$$

Then the idea control law can be designed as

$$
\delta_{z a}=\frac{1}{a_{25}}\left\{-a_{24} \alpha-a_{22} \omega_{z}-k_{a 1} e_{\omega}-k_{a 2} \frac{e_{\omega}}{\left|e_{\omega}\right|+\varepsilon_{a}}-f_{2}-\dot{f}_{1}+\dot{\omega}_{z a}^{d}\right\}
$$

Here we use the value $\dot{x}_{1}$ as the value of $\dot{\omega}_{z a}^{d}$ since $x_{1} \rightarrow \omega_{z a}^{d}$. And assume $-f_{2}-\dot{f}_{1}$ is bounded, then there exists $k_{a 2}$ such that $\left|-f_{2}-\dot{f}_{1}\right| \leq k_{a 2}$. Also consider that $k_{a 2} \frac{e_{\omega}}{\left|e_{\omega}\right|+\varepsilon_{a}} \approx k_{a 2} \operatorname{sgn}\left(e_{\omega}\right)$, so we design the real control law as

$$
\delta_{\text {za }}=\frac{1}{a_{25}}\left\{-a_{24} \alpha-a_{22} \omega_{z}-k_{a 1} e_{\omega}-k_{a 2} \frac{e_{\omega}}{\left|e_{\omega}\right|+\varepsilon_{a}}+\dot{x}_{1}\right\}
$$

Then if we choose a Lyapunov function as

$$
V=\frac{1}{2} e_{\alpha}^{2}+\frac{1}{2} e_{\omega}^{2}
$$

And its derivative can be calculated as

$$
\dot{V} \leq-k_{\alpha 1} e_{\alpha}^{2}-k_{a 1} e_{\omega}^{2}
$$

So according to the Lyapunov stability theorem, the whole system is stable.

\section{Numerical Simulations}

The air coefficient of a type of missiles can be set as

$$
a_{25}=-167.87 ; a_{35}=0.243 ; a_{22}=-2.876 ; a_{24}=-193.65 ; a_{34}=1.584
$$

It is a kind of non-mini-phase system. Set control parameters as

$$
R=10 ; k_{a 1}=8 ; k_{a 2}=15 ; k_{\alpha 1}=20 ; k_{\alpha 2}=10
$$

Then simulation results can see below figures 1-5. Figure 1 to Figure 3 shows the missile system is stable and attack angle can trace to the desired value 5 degree. And figure 4 and figure 5 shows that the estimation of angle speed and its derivative. So it can provide derivative information for the backstepping design method. 


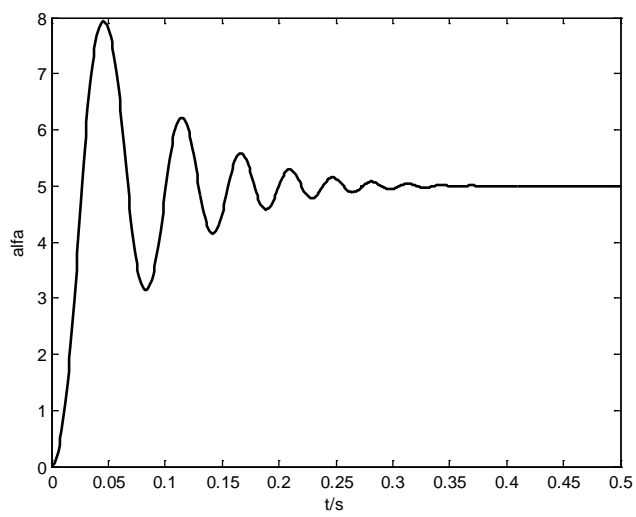

Figure 1 The curve of attack angle

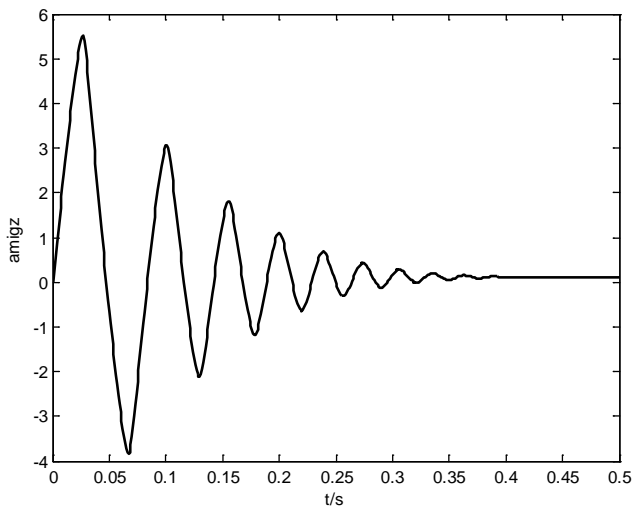

Figure 2 The figure of angle speed

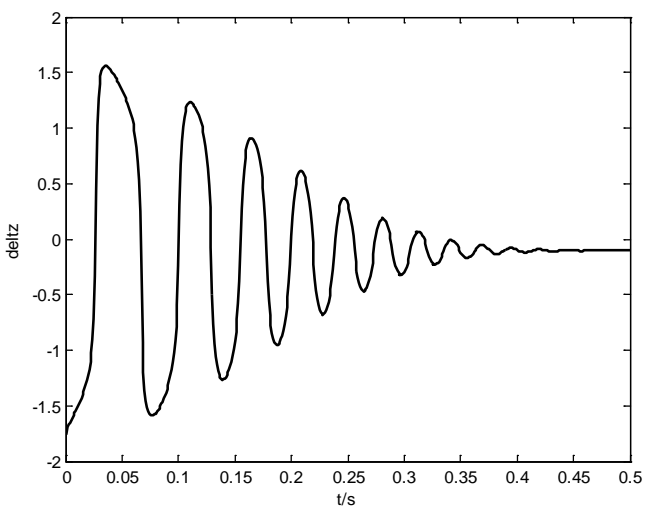

Figure 3 The curve of actuator

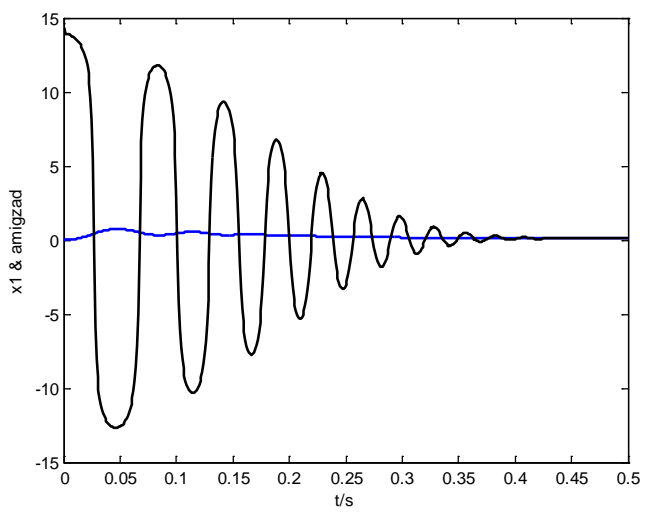

Figure 4 The estimation of $\omega_{z a}^{d}$ 


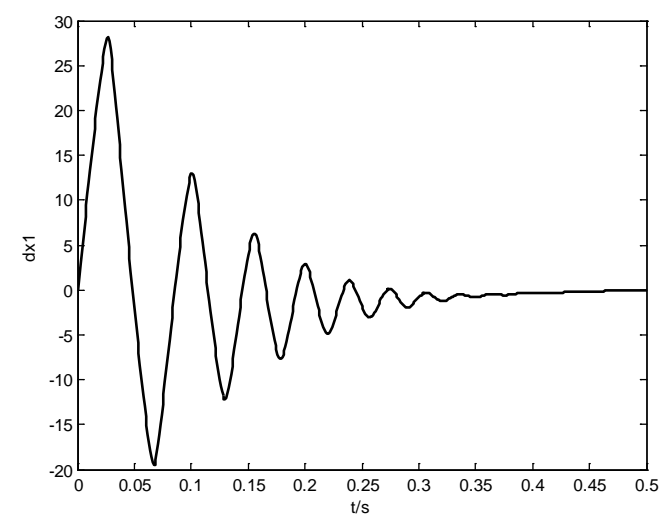

Figure 5 The state of $\dot{\omega}_{z a}^{d}$

\section{Conclusions}

A novel tracking differentiator based backstepping method was proposed and applied in the pitch channel missile system to make the attack angle tracking to the desired value. Also a Lyapunov function method was used to prove the stability of the whole system. And detailed simulation were done to test and check the stability of proposed control strategy.

\section{Reference}

[1] Jason J G, Kathryn W J, et al. A simplified adaptive robust back-stepping approach using sliding modes and a z-swapping identifier[C]. Proceedings of the American Control Conference, Denver,2003.

[2] Zhou Y, Wu Y, Hu Y. Robust backstepping sliding mode control of a class of uncertain MIMO nonlinear systems[C]. 2007 IEEE International Conference on Control and Automation, Guangzhou, CHINA, 2007.

[3] Lee T,Kim Y. Nonlinear adaptive flight control using back-stepping and neural networks controller [J]. Journal and Guidance,Control and Dynamics,2001,24( 4) : 675-682.

[4] Zhu Kai,Qi Naiming, Qin Changmao. Adaptive sliding mode controller design for BTT missile based on back-stepping control[J]. Journal of Astronautics, 2010, 31( 3) : 769-773.

[5] Chen Y, Dong C Y, Wang Q, et al. Reaction-jet and aerodynamics compound control missile autopilot design based on adaptive fuzzy sliding mode control via backstepping[J]. Acta Aeronautica et Astronutica Sinica, 2007, 28(SI): 1141-145.

[6] Jay F, Manu S, Marios P, Backstepping based flight control with adaptive function approximation[J]. Journal of guidance, Control, and Dynamics, 2007, 30(2):322-336.

[7] Tsung-Ying Chiang, Jui-Sheng Lin Teh-Lu Liao, Jun-Juh Yan, Antisynch-ronization of uncertain unified chaotic systems with dead-zone nonlinearity, Nonlinear Analysis 68 (2008) 2629-2637

[8] May R M. Simple mathematical models with very complicated dynamics [J], Nature, 1976, 261: 459-462. 